The International Journal Of Engineering And Science (IJES)

|| Volume || 6 || Issue || 1 || Pages || PP 40-42 || 2017 ||

ISSN (e): $2319-1813 \operatorname{ISSN}$ (p): $2319-1805$

\title{
A Model of Decision Support System for Research Topic Selection and Plagiarism Prevention
}

\author{
Dr. Hardik B. Pandit ${ }^{1}$, Dr. Dipti B. Shah ${ }^{2}$ \\ ${ }^{1,2}$ G H Patel Post Graduate Department of Computer Science, Sardar Patel University Near Jain Derasar, Nana \\ Bazar, Vallabh Vidyanagar, Gujarat, India.
}

\begin{abstract}
The paper proposes a model of the decision support system for deciding a research topic in academia. The biggest challenge for a student in the field of research is to identify area and topic of research. The paper explains the model which helps student to identify the most suitable area and/or topic for academic research. The model is also design to assist supervisors to explore latest areas of research as well as to get rid of non intentional plagiarism. The model facilitates the user to select either keyword bases topic search or questionnaire based topic search. The model uses local database and service of a Meta search engine in decision making activity.
\end{abstract}

ABSTRACT

Keywords: Decision Support System, Meta Search Engine, Plagiarism, Research Topic Selection

Date of Submission: 11 January $2016 \quad$ Date of Accepted: 26 January 2017

\section{INTRODUCTION}

Decision Support Systems are the systems which assist the human being in decision making activity [8]. Proposed model for decision support system is for assistance in making choice of research topic for academic research. The model combines the approaches of decision support system and web content mining to decide the most suitable area of research for a particular student. The student answers the questions asked by the system in terms of predefined key-words. Based on these answers, the model searches the content from various Websites and presents most relevant research topics on which researchers are working. The student can go through these topics and can decide his/her own research topic. The supervisor uses the system to explore the latest areas of research in his/her field by using explore facility. By entering a key word he/she can get maximum possible links related to the area of research. The user of this system can also search the related research work done in the same field through the feature provided by the system. This feature prevents user from non-intentional plagiarism.

\section{LITERATURE REVIEW}

In context of selecting research topic in the academia, especially for Ph.D., some authors have done significant work. Li Yu, Jie Yang, Dong Yang, and Xiaoping Yang have explained a research topic decision support system to help a user to quickly find potential research topic based on iteratively paper recommendation. This system suggests appropriate research literature to the user, based on his/her preferences given to the system. The user reads this literatures/ research papers and gets his/her ideas clear about the respective topic of research. The key focus of the system is to provide the most appropriate literature in each round, so he can quickly find the research topic while spending minimal time. [1] Iyigün, M. Güven has also worked on the DSS for R\&D project selection and resource allocation under uncertainty. A framework and model of a Decision Support System (DSS) is presented as an effective tool to help choose projects using complex criteria such as risk, cost, decision hierarchies, as well as attempting to meet profit, human resources, and budget objectives. The DSS is comprised of three phases which are divided into modules: Screening and Scoring, Assessment, and Resource Allocation, which uses the PROTRADE method. [2] This model supports the selection process for the research and development project by the managers. A research work is also done and hosted in Purdue University, Indiana regarding selection of research topic in the field of computer science. The tool is named as "A CS Research Topic Generator" or "How To pick A Worthy Topic In 10 Seconds". The system displays different phrases in the field of computer science in three columns which contain 21 entries in each. To generate a technical phrase, user should randomly choose one item from each column. For example, selecting synchronized from column 1 , secure from column 2, and protocol from column 3 produces: "A synchronized secure protocol". Then, two phrases can be combined with simple connectives, making the result suitable for the most demanding use. Possible connectives include: for, related to, derived from, applied to, embedded in, etc. For example, one could generate a thesis title by selecting a second phrase and a connective: "A synchronized secure protocol for an 
interactive knowledge-based system". After selection of such topic, the system also offers searching facility for relevant literature on a search engine. [3] University of Michigan- Flint has also guided students in deciding the research topic for their doctoral research work. They have suggested following steps for the purpose to be performed in sequence: brainstorm for ideas, choose a topic that will enable you to read and understand the literature, ensure that the topic is manageable and that material is available, make a list of key words, be flexible, define your topic as a focused research question, research and read more about your topic, formulate a thesis statement. [4] In addition to above mentioned literature, the web sites of Massachusetts Institute of Technology, University of Buffalo, and University of Illinois Urbana-Champaign are also studied for guidelines of selecting a research topic.[5][6][7] To supplement the survey of literature, blogs are also studied in context of selection of research topic.

\section{LIMITATIONS OF EXISTING SYSTEMS}

After analysing the existing systems from different aspects, following limitations are found:

i. The system proposed by Yu Li, Yang Jie, Yang Dong, and Yang Xiaoping focuses on display of related research papers in the particular filed selected by student. It shows most appropriate literature to the student. Student will have to judge that which is the most relevant, to decide the research topic. Moreover, the system depends on its database for suggesting related literature. This imposes limitation that only those research papers which are stored in the database could be displayed to the student. Anything outside of database is not accessible to the student if it is not updated regularly.

ii. The paper by Iyigün, M. Güven explains the idea of selection of research topic but it is not centred on academic research. Though, the paper presents very good ideas about risks, cost, division hierarchies, profit-loss, human resources, and budgetary aspects of research project, it is more towards industrial research and development.

iii. "A CS Research Topic Generator", by Purdue University is a good system to generate research topic, but it does not allow student to consider anything other than given keywords. Moreover, the system does not assist the student in making decision of area of research.

iv. Various reputed universities have explained about deciding research topic for academic research, but their implementation is upon the interpretation of the student. Wrong or partial understanding of such suggestions may mislead the student.

\section{SCOPE OF RESEARCH}

Considering limitations of the current systems for deciding research topic for academic research, one needs a decision support system which can overcome the limitations mentioned in section 3. A computerized decision support system is required to assist the student in deciding the most suitable area as well as topic of research. The automatic system should understand the attitude of the student based on interaction and should present maximum possible areas and topics of research from the World Wide Web.

\section{PROPOSED MODEL}

To overcome the limitations mentioned in section 3, and to assist the research student to select the most suitable research topic, a model is proposed. Fig. 1 shows the proposed model which is under implementation.

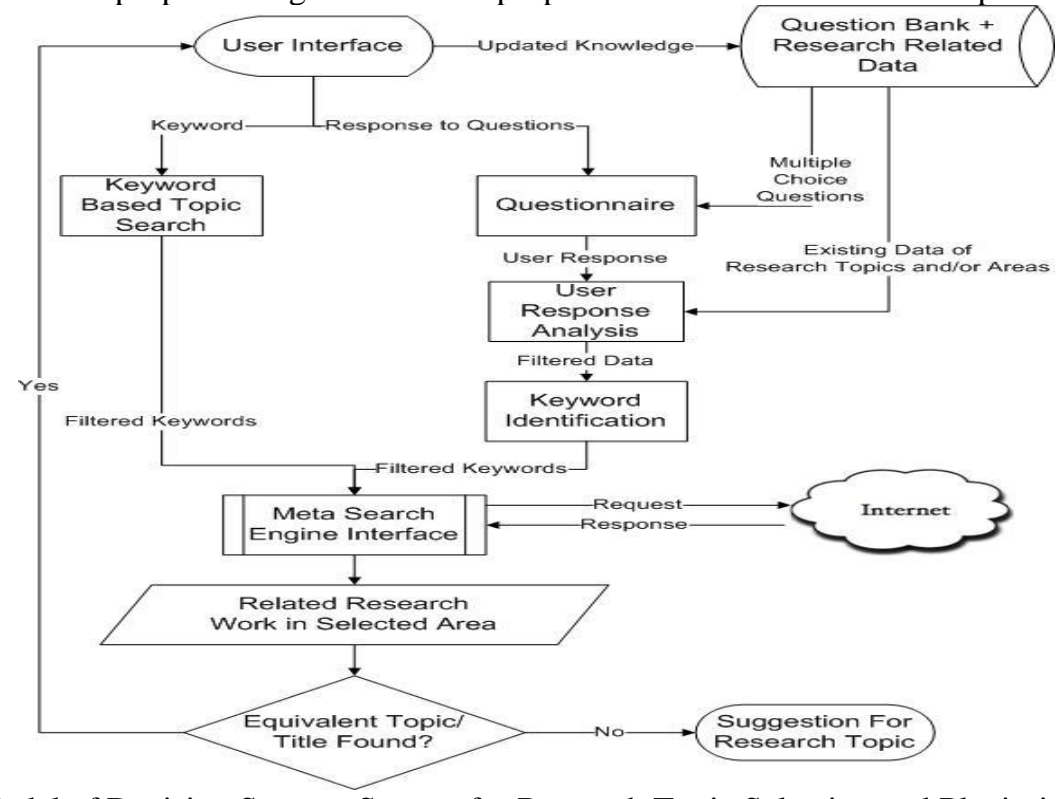

Figure 1: Model of Decision Support System for Research Topic Selection and Plagiarism Prevention 
The model operates in following steps:

i. User Interface: The users of the system are the student who is searching for research area/topic, the supervisor who want to get updated with latest trends of research, and administrator who is responsible to manage question bank and web services. The user interfaces are separate for all the three users.

ii. Questionnaire: Questionnaire is the collection of common questions regarding research attitude of the student. Initial questions are common and generalized, but as student progresses in answering, the questions become more specific. Consequently, the questions focus on narrow area of research to find details of student's attitude.

The questionnaire consists of such questions whose answers could be given within maximum three keywords. For this, multiple choice questions will be given to the student.

iii. User Response Analysis: The answers to the questionnaire, given by the student are analyzed to extract the attitude of the student for the particular area of research. Repeatedly occurring keywords are identified and saved for further processing.

iv. Keyword Identification: keywords identified in previous step are processed to filter them. The keywords are filter to identify unique meaning. Ambiguous keywords are processed and replaced by appropriate word.

v. Keyword Based Topic Search: This is an interface for the student as well as the supervisor to search the topic for research. If the student has clear idea about area of research but not able to find good topic, this option will help him to find research topic. On the other hand, if the supervisor wants to know genuineness of the research topic submitted by student he/she can verify it by this facility.

vi. Meta Search Engine Interface: The system provides an interface to any of the popular Meta search engines to search for the keywords derived from earlier steps on the internet. Instead of providing results from single search engine, the system uses service of Meta search engine to enlarge the scope of search of the topic. The results are displayed to the user in terms of related research work in selected field.

vii. As a last step of the model, user can see the current status of the research topic, i.e. whether the preferred topic is indeed new or research is going on somewhere else on the same topic. If topic is not feasible to work with, because of any reason, the user can repeat the procedure with new set of keywords.

\section{CONCLUSION}

The proposed model is applicable to the academic community in various ways, as mentioned below:

i. To assist students in selection of appropriate research topic: If the student is sure about the area of research, he/she can search various research topics in the same area by searching based on key word. On the other hand, if the student is not sure about research area, by carefully answering the questionnaire he/she can get suggestion of appropriate research topic.

ii. To assist supervisors in exploring latest areas of research: Supervisors are supposed to be updated in their field of research. The Meta search engine interface provides the ease of searching latest trends in research in less time than ordinary search on regular search engines.

iii. To prevent non-intentional plagiarism: The model can be used to search the work done in relevant research area. Therefore, student or supervisor can avoid selecting existing research work.

iv. To provide exposure of research work done in the university: The model also serves as an interface between research data of university and users. One can search the research work going on in the university to get assistance in his/her own work.

\section{REFERENCES}

[1]. Yu, Li; Yang, Jie; Yang, Dong; and Yang, Xiaoping, "A Decision Support System for Finding Research Topic based on Paper Recommendation" (2013). PACIS 2013 Proceedings.190. http://aisel.aisnet.org/pacis2013/190

[2]. Iyigün, M. Güven, "A Decision Support System For R\&D Project Selection And Resource Allocation Under Uncertainity", Project Management Journal, December 1993

[3]. A CS Research Topic Generator, Purdue University

[4]. https://www.umflint.edu/library/how-select-research-topic

[5]. http://libguides.mit.edu/select-topic

[6]. http://library.buffalo.edu/help/research-tips/topic/

[7]. http://guides.library.illinois.edu/choosetopic

[8]. Hardik Pandit, Dipti Shah, "Image Processing and Analysis for Medical Palmistry", Lambert Academic Publishing, ISBN: 978-3659-12268 